\title{
A proposed EEG study: the role of object affordance during action observation
}

\author{
Rebecca Hailperin-Lausch, Isaiah Inns, Elizabeth DeSilva
}

Faculty Mentor: Dr. Bennett Bertenthal, Department of Psychological and Brain Sciences Indiana University

\section{ABSTRACT}

Over the past two decades, research on the human mirror neuron system (MNS) has flourished. According to this model, there is substantial evidence that both action execution and action observation activate the motor system. However, to date, few studies have attempted to examine the role that object affordance may have during action observation. The proposed study attempts to assess this and other issues by having participants watch videos of an actor making goal-directed reaches to a common household object. In the congruent condition, the actor makes a reach and grasps the handle of a mug. In the affordance incongruent condition, the actor makes a reach but grasps the side of the mug opposite from the handle. Electroencephalogram (EEG) will be recorded throughout participant viewing and the EEG data will be decomposed into frequency bands using a Morlet wavelet analysis. The mu rhythm $(8-13 \mathrm{~Hz})$ will be of particular interest. Electrode sites of interest include sites over the central parietal areas as well as frontal sites. It is hoped that the proposed study will provide insight into the role of object affordance during action observation.

\section{KEYWORDS: Action Observation, Object Affordance, Mirror Neuron System, Mu Rhythm}

\section{INTRODUCTION}

$\mathrm{E}$ vidence suggests that similar patterns of brain activation occur during both action execution and action observation. This model, often known as the mirror neuron system (MNS), is supported by evidence from studies done in macaque monkeys (Di Pellegrino et al. 1992, Rizzoliti et al. 1996), behavioral studies (Brass, Bekkering, Wohlschläger, \& Prinz 2000, Bertenthal, Longo, \& Kosobud 2006, Longo, Kosobud, \& Bertenthal 2008, Gillmeister et al. 2008, Boyer \& Bertenthal 2013) and studies done using fMRI (Buccino et al. 2001, Grèze, Armony, Rowe, \& Passingham 2003). It is thought that the mirror neuron system helps us to identify the intentions of others (Iacoboni et al. 2005) and may facilitate empathy (Iacoboni 2009).

Recent research indicates that event related desynchronization of the mu rhythm $(8-13 \mathrm{~Hz})$ may reflect activation of the mirror neuron system. For instance, Muthukumaraswamy, Johnson, and McNair (2004) observed that participants showed mu suppression when viewing a grasp of a manipulandum but not when viewing a similar, empty grasp. In a different study, Perry and Bentin (2009) detected mu desynchronization when participants viewed reaches but not when participants viewed other movements.

Not only has been mu desynchronization been observed during action observation, but also when viewing tools and objects. For instance, Proverbio (2012) had participants view objects with a congruent grasp as well as non-objects and objects with an incongruent grasp. Participants showed significantly more mu suppression when viewing objects with congruent grasps than during the other conditions. In a similar, behavioral study, Bach, Bayliss, and Tipper (2010) had participants move a joystick to the left or right while watching an actor make grasps that were either congruent or incongruent with the affordance of an object. Importantly, the compatibility effects were only found when participants viewed grasps that were congruent with the affordance. These findings suggests that the perception of objects may be important for motor activation during action observation.

When we observe someone performing a goal-directed action, we pay attention to not only the individual, but also the object that they are interacting with. In his theory of object affordance, Gibson (1979) proposed that the properties of certain objects naturally allow us to act on them. For instance, we typically pick up a mug using the handle rather than using the top or the side opposite from the handle. The handle affords a grasping motion allowing us to easily pick up the mug.

Relatively few studies have attempted to dissociate the roles that object affordance might play in action observation. Recently, Bach, Nicholson, and Hudson (2014) proposed the affordance-matching hypothesis. According to this model, individuals automatically retrieve information about the objects around themselves as well as the objects around others. This model suggests that understanding the properties of objects and the ways that they can be manipulated are essential during action observation. Thus, the purpose of the current is to assess this relationship between object affordance and action observation by measuring event related desynchronization of the mu rhythm.

\section{PROPOSED METHODS}

\section{Participants}

We hope to test approximately 30 participants all of whom will be right-handed with normal or corrected-to-normal vision. They will be recruited from a large, public university and will receive course credit for participation in the study.

\section{Design}

The stimuli consists of eight reach videos. In the videos, an actor sits behind a table in the center of the screen. A 
red mug with a handle is seen on either the left or the right side of the table, depending on the condition. All reaches are right handed only and both ipsilateral and contralateral reaches are done. In the congruent condition, the actor reaches for the handle of the cup. In the incongruent condition, the actor reaches for the side of the cup opposite of the handle. These same 4 conditions are also done with an inverted cup. Examples from each condition can be found in figure 1. To ensure the best possible viewing conditions, the contrast of the stimuli was adjusted in Adobe Premiere Pro. Additionally, Adobe Premier Pro was used to ensure that all reaches had the same number of frames. One to three frames were systematically removed. All reaches lasted 1.2 seconds.

Stimuli will be presented using EPRIME 2.0. Participants will sit 70 inches away from a 17" computer monitor. Each trial will consist of a fixation cross for $500 \mathrm{~ms}$ and one reach which will be paused after the completion of the reach. The still frame of the reach will remain on the screen for $1000 \mathrm{~ms}$ and the intertrial interval (ITI) will be $1000 \mathrm{~ms}$. The study will be divided into 8 blocks which will last approximately 5 minutes. In each block, participants will see 48 trials where each of the eight conditions are presented six times per block. Thus, participants will view a total of 384 trials which are pseudorandomized within the blocks. Continuous EEG data will be collected throughout the duration of the stimulus presentation.

\section{Procedure}

Participants will be instructed to avoid excessive movements and to blink during the ITI when possible. There will be 3 probe trials per block of 48 that are interspersed randomly. Participants will be instructed to press the space bar when a white cross appears on the cup. These probe trials are used to ensure participants pay attention throughout the task. EEG data for the probe trials will not be analyzed.

\section{EEG Recording, Preprocessing, and Analysis}

Participants will wear the Hydrocel geodesics sensor net which contains 64 active $\mathrm{Ag} / \mathrm{AgCl}$ electrodes. Data will be amplified using EGI Net Amp 300 and will be sampled at $1000 \mathrm{~Hz}$. Electro-oculogram (EOG) will also be recorded in order to detect eye movements. Impedance measures below $50 \mathrm{k} \Omega$ are considered acceptable.

Data will be processed using the EEGLAB toolbox in Matlab. The continuous data will be band-passed filtered between 0.1 and $100 \mathrm{~Hz}$. If $60 \mathrm{~Hz}$ line noise is present, the function cleanline will be used to remove this noise. The data will be epoched into 3.2 second epochs. The epoched data will be visually inspected for bad channels and gross muscle artifacts which will be removed. Participants will not be used in the final sample if more than $33 \%$ of their total trials have to be removed. Independent component analysis (ICA) will be run and components that constitute eye blinks, eye movements, or EMG will be removed. Data will be rereferenced offline to the average of all scalp electrodes. Any bad channels that had been removed previously will be interpolated. After pre-processing, data will be analyzed using the EEGLAB toolbox. A Morlet wavelet analysis will be run to decompose the signal into frequency bands.

\section{EXPECTED RESULTS}

The proposed study is in the process of being run and thus no data is currently available. Since participants only viewed right-handed reaches, it is predicted that the effect will be lateralized to the left hemisphere. There are also several other hypotheses that are being considered.

It could be that the participants are mapping the action of the actor onto their motor system, suggesting activation of the mirror neuron system. If this is the case, we might expect to see greater desynchronization of the mu rhythm in the affordance congruent condition for both ipsilateral and contralateral reaches than in the affordance incongruent condition. Another possibility is that the affordance of the

Figure 1. Still frames taken from stimuli: top row is the upright condition and bottom row is the inverted condition.
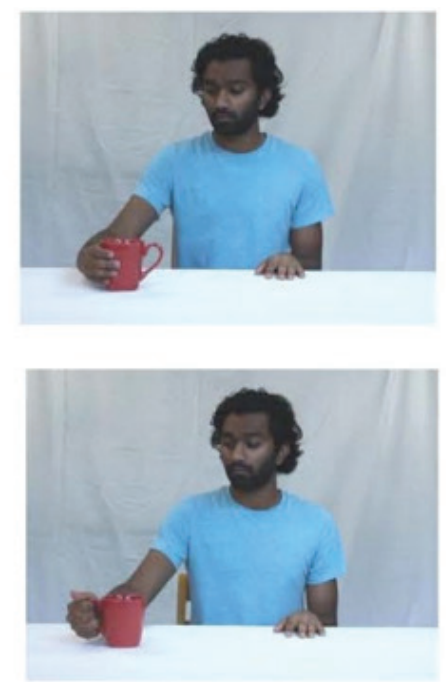
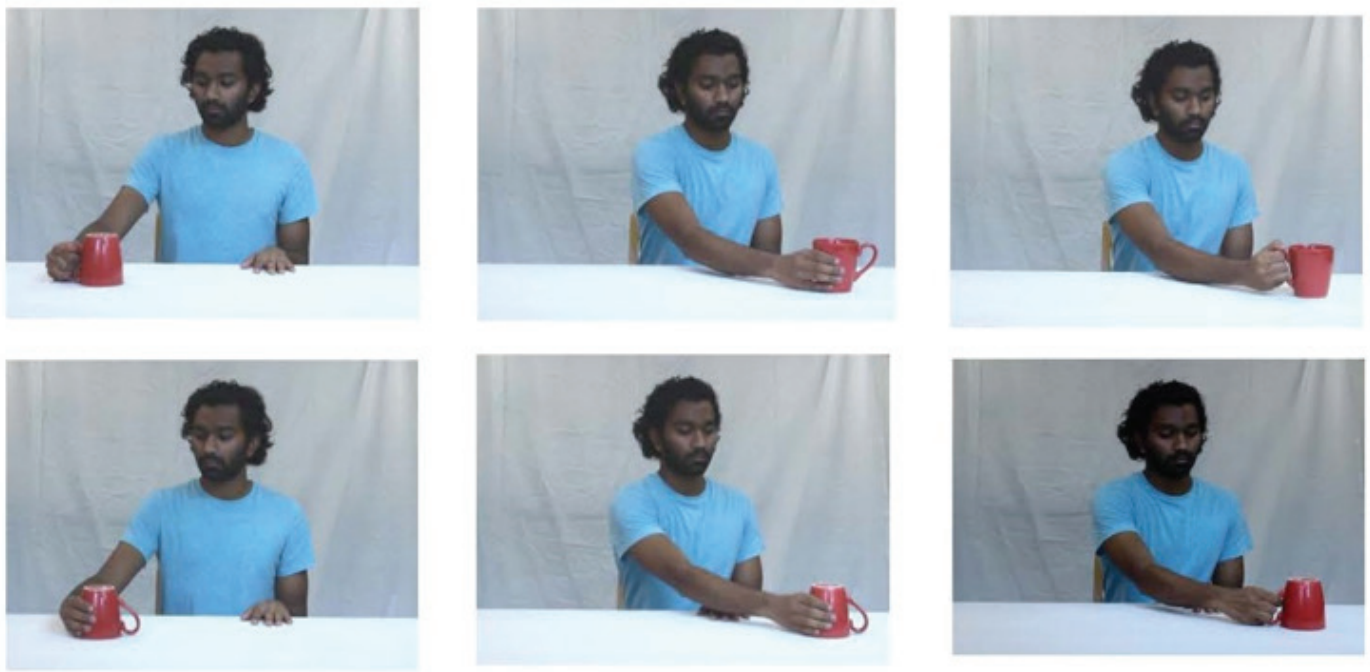
object does not affect our ability to activate the mirror neuron system. If this is the case, then we would expect to see similar patterns of mu desynchronization in both the inverted and the affordance incongruent condition as in the affordance congruent condition because all reaches would activate the mirror neuron system regardless of the affordance of the object.

One of the advantages of using the Morlet wavelet analysis is that both the time domain and frequency domain can be assessed. Thus, it also possible to look at latency differences in desynchronization. It seems plausible that participants may be faster to map the motor representation of the reach during the congruent condition, a normal, everyday action, than they would be to map the motor representation of the reaches to the incongruent condition or to reaches towards the inverted cups. Thus, suppression may be observed later during these conditions than in the congruent condition.

\section{DISCUSSION AND CONCLUSION}

This study has several advantages over previous studies. First, this study differs from previous studies in that it examines action observation during passive viewing rather than during a task which eliminates any possible confounds that could occur from activation of the motor system. In addition, instead of viewing still images of a completed action, participants in the current study watch the entire action. Finally, the participants view the entire upper body of the actor when performing a goal directed action. In much of literature focusing on object affordances and action observation, participants typically see the arm and hand of the actor but not the face or torso. Thus, the proposed study is more similar to what participants observe in daily life.

The current study hopes to disentangle the hypotheses mentioned above while also providing further insight into how action observation and object affordance are related. This study may provide further evidence for the idea that the mu rhythm can be used as an index of the mirror neuron system. Finally, the current study may provide important ideas for further research.

\section{REFERENCES}

Bach, P., Bayliss, A. P., \& Tipper, S. P. (2010). The predictive mirror: interactions of mirror and affordance processes during action observation. Psychonomic bulletin \& review, 18(1), 171-176.

Bach, P., Nicholson, T., \& Hudson, M. (2014). The affordance-matching hypothesis: how objects guide action understanding and prediction. What can we make of theories of embodiment and the role of the human mirror neuron system?, 99.

Bertenthal, B.I., Longo, M. R., \& Kosobud, A. (2006). Imitative response tendencies following observation of meaningless actions. Journal of Experimental Psychology: Human Perception \& Performance, 32, 210-225.
Boyer, T. W., \& Bertenthal, B. I. (2013). Covert and overt social attention: Pointing automatically orients direction of attention, Journal of Vision, 13(9), 1128; doi: 10.1167/13.9.1128.

Brass, M., Bekkering, H., Wohlschläger, A., \& Prinz, W. (2000). Compatibility between observed and executed finger movements: comparing symbolic, spatial, and imitative cues. Brain and cognition, 44(2), 124-143.

Buccino, G., Binkofski, F., Fink, G. R., Fadiga, L., Fogassi, L., Gallese, V., ... \& Freund, H. J. (2001). Action observation activates premotor and parietal areas in a somatotopic manner: an fMRI study. European journal of neuroscience, 13(2), 400-404.

Di Pellegrino, G., Fadiga, L., Fogassi, L., Gallese, V., \& Rizzolatti, G. (1992). Understanding motor events: a neurophysiological study. Experimental brain research, 91(1), 176-180.

Gibson, J. J. (1979). The ecological approach to visual perception. Boston: Houghton Mifflin.

Gillmeister, H., Catmur, C., Liepelt, R., Brass, M., \& Heyes, C. (2008). Experience-based priming of body parts: a study of action imitation. Brain research, 1217, 157-170.

Grèzes, J., Armony, J. L., Rowe, J., \& Passingham, R. E. (2003). Activations related to "mirror" and "canonical" neurones in the human brain: an fMRI study. Neuroimage, 18(4), 928-937.

Iacoboni, M. (2009). Imitation, empathy, and mirror neurons. Annual review of psychology, 60, 653-670.

Iacoboni, M., Molnar-Szakacs, I., Gallese, V., Buccino, G., Mazziotta, J. C., \& Rizzolatti, G. (2005). Grasping the intentions of others with one's own mirror neuron system. PLoS Biol, 3(3), e79.

Longo, M. R., Kosobud, A., \& Bertenthal, B. I. (2008). Automatic imitation of biomechanically impossible actions: Effects of priming movements vs. goals. Journal of Psychology: Human Perception and Performance, 34, 489-501.

Muthukumaraswamy, S. D., Johnson, B. W., \& McNair, N. A. (2004). Mu rhythm modulation during observation of an object-directed grasp. Cognitive Brain Research, 19(2), 195-201.

Perry, A., \& Bentin, S. (2009). Mirror activity in the human brain while observing hand movements: A comparison between EEG desynchronization in the $\mu$-range and previous fMRI results. Brain research, 1282, 126-132.

Proverbio, A. M. (2012). Tool perception suppresses 10-12Hz $\mu$ rhythm of EEG over the somatosensory area. Biological psychology, 91(1), 1-7.

Rizzolatti, G., Fogassi, L., \& Gallese, V. (2001). Neurophysiological mechanisms underlying the understanding and imitation of action. Nature Reviews Neuroscience, 2(9), 661-670.

Rizzolatti, G., Fadiga, L., Gallese, V., \& Fogassi, L. (1996). Premotor cortex and the recognition of motor actions. Cognitive brain research, 3(2), 131-141. 
